\title{
Intragroup differences in COVID-19 vaccine attitudes among Black Americans
}

\author{
Charles R. Senteio ${ }^{1,2^{*}}$, Christie Newton ${ }^{3}$, Gordon Pennycook ${ }^{3}$, David G. Rand ${ }^{1}$
}

${ }^{1}$ Sloan School of Management, Massachusetts Institute of Technology, ${ }^{2}$ Rutgers University School of Communication and Information, ${ }^{3}$ University of Regina, Hill/Levene Schools of Business

Keywords: COVID-19 Racial Inequity, COVID-19 Perceptions, COVID-19 Vaccination, African American Acculturation

This working paper has not yet been peer reviewed; posted 4-6-2021

COVID-19 vaccine hesitancy among Black Americans threatens to further magnify racial inequities in COVID-19-related health outcomes that emerged in the earliest stages of the pandemic. Here we shed new light on attitudes towards COVID-19 vaccines by considering intragroup variation. Rather than analyzing Blacks as a homogenous group, we examine the relationship between COVID-19 vaccine attitudes and the extent to which participants are aligned with African American versus White culture (i.e., level of "acculturation"). In a sample of $N=997$ Black Americans, we find that stronger alignment with African American culture predicts substantially more negative attitudes towards COVID-19 vaccination, above and beyond variation explained by age, gender, education, and socioeconomic status. This relationship was substantially attenuated when controlling for suspicion of the healthcare system, but not perceptions that healthcare system treats Blacks unfairly, science knowledge, or cognitive reflection. The intragroup differences among Blacks in COVID-19 vaccine perceptions uncovered here provide insights into designing interventions that provide health information that targets the relevant factors for vaccine hesitancy in differing subgroups.

Racial inequities for COVID-19 infection, hospitalization, and mortality were reported across the U.S. early in the COVID-19 pandemic, with Black individuals having particularly high risk. ${ }^{1,2}$ Blacks also showed high levels of COVID-19 vaccine hesitancy well before vaccines became available, ${ }^{3}$ and the proliferation of misinformation increased vaccine hesitancy among Blacks compared to other racial minority groups. ${ }^{4}$ Higher negative attitudes towards COVID-19 vaccines among Blacks are particularly concerning as they threaten to further amplify racial inequities in COVID-19 outcomes.

Here, we seek to shed new light on these negative attitudes about COVID-19 vaccines among Black Americans. We do so by addressing a widespread shortcoming of research on health inequality and COVID-19: analyzing Black Americans as a homogenous group. Intragroup variation in COVID-19 vaccine attitudes have been ignored, despite a wealth of evidence for important cultural variation among Blacks that influence perceptions and subsequent health behaviors. ${ }^{5,6}$ To the extent that attitudes towards COVID-19 vaccines vary 
substantially across different segments of the Black community, this has important implications for which groups to target with pro-vaccine messaging, and what messages are most likely to be effective.

To gain insight into intragroup cultural variation, we examined the extent to which participants are aligned with African American culture (i.e., have a "traditional" versus "acculturated" cultural orientation) using the revised African American Acculturation Scale (AAAS-R). ${ }^{6}$ The AAAS-R measures eight theoretically derived dimensions of African American culture: 1) agreement with traditional African American religious beliefs and practices; 2) preference for Black music, magazines, and people; 3) agreement with traditional attitudes among Black Americans about Whites and about racism; 4) participation in traditional African American family practices; 5) agreement with traditional African American family values; 6) agreement with traditional African American health beliefs and practices; 7) agreement with traditional African American cultural superstitions; and 8) the extent to which one grew up and currently resides in a mostly Black neighborhood.

Acculturation has been shown to correlate with a wide variety of perceptions and health outcomes for Black Americans, including food-related attitudes and dietary behavior, ${ }^{7}$ perceptions of social support and suicidal ideation, ${ }^{8}$ distrust for Whites and knowledge of AIDS transmission, ${ }^{9}$ and cigarette smoking. ${ }^{10}$ Acculturation scales developed for other racial minority and immigrant groups have also been shown to be predictive of a wide range of health behaviors. $^{11}$

Given the previously documented associations between acculturation and other health perceptions and behaviors, we examine the relationship between acculturation and negative attitudes towards COVID-19 vaccines in a large, demographically representative sample of $N=997$ Black Americans. We also used the Group-Based Medical Mistrust Scale ${ }^{12}$ to investigate the relationship with mistrust of the medical system - which is more pronounced among Blacks compared to Whites ${ }^{13}$ - and the extent to which such mistrust explains acculturation-based variation in COVID-19 vaccine attitudes. Finally, we examine relationships with science knowledge and cognitive reflection (the tendency to think analytically rather than relying on one's intuitions), which have been found to predict COVID-19 vaccine attitudes in general population samples. ${ }^{14}$ All of our analyses include controls for age, gender, education, and socioeconomic status.

\section{Results}

We find a significant negative correlation between acculturation and negative attitudes towards the COVID-19 vaccine $(\beta=-.227, \mathrm{t}=8.03, \mathrm{p}<.001)$. As shown in Figure 1 , participants who were more aligned with African American culture had substantially more negative COVID19 vaccine attitudes. To help contextualize the size of this relationship, acculturation explained $35.2 \%$ as much variation in vaccine attitudes as age, 3.9 times more variation than gender, 4.0 times more variation than education, and 7.0 times more variation than socio-economic status. Importantly, excluding the Health Beliefs \& Practices subscale had little effect on the relationship between acculturation and negative vaccine attitudes $(\beta=-.214, t=7.54, p<.001)$, and the relationship also held when considering each of the eight subscales separately $(\mathrm{p}<.001$ for all). 


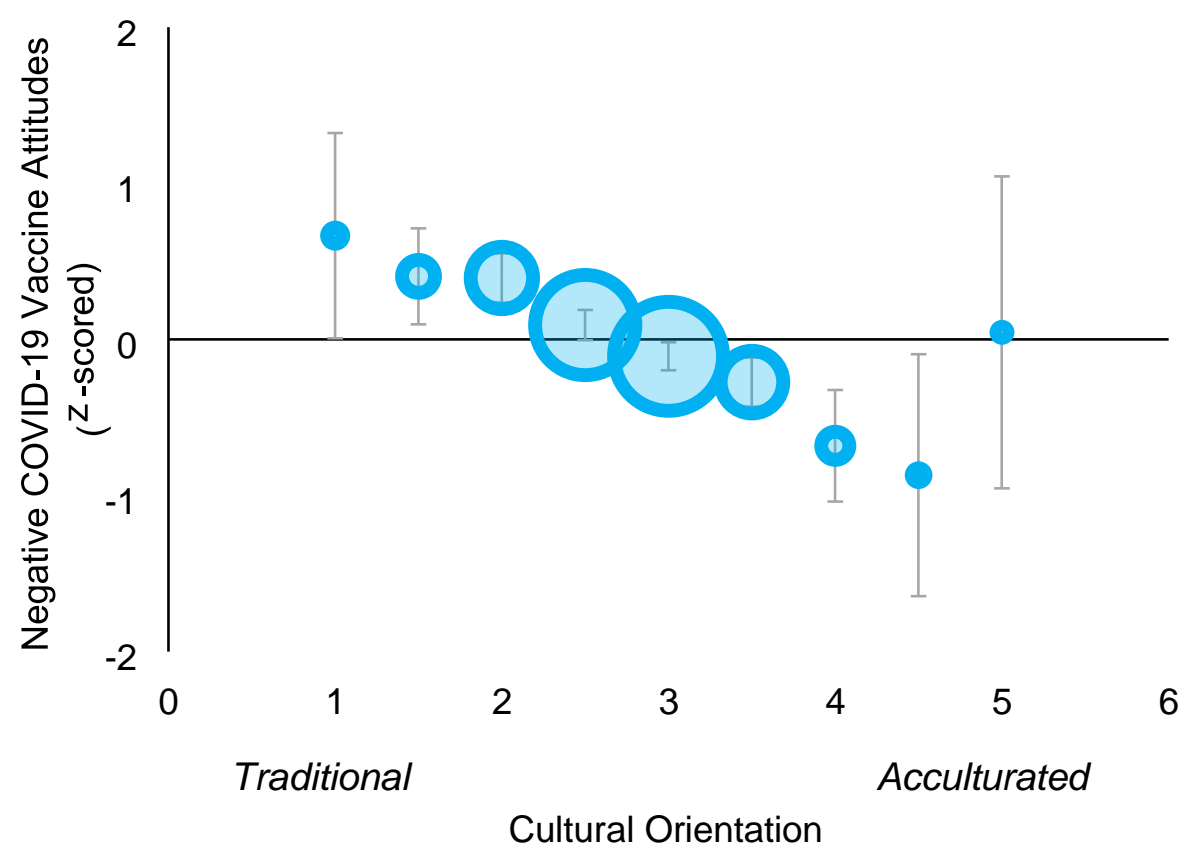

Figure 1. Negative attitudes towards COVID-19 vaccines (z-scored) based on level of acculturation; partialling out variation explained by age, gender, education, and socio-economic status. Dot size proportional to number of observations. Error bars indicate $95 \%$ confident intervals.

To provide some evidence of a causal effect of culture on COVID-19 vaccination attitudes, we randomized the order of presentation of the acculturation measure versus the COVID-19 vaccine attitudes measure. Consistent with a causal effect, we find a significant interaction between acculturation and presentation order (both when only interacting order with acculturation, $\mathrm{t}=2.74, \mathrm{p}=.006$, and when also interacting order with each control variable, $\mathrm{t}=2.12$, $\mathrm{p}=.034)$, such that the relationship between acculturation and vaccine attitudes was twice as large when asked about acculturation first $(\beta=-0.30, \mathrm{t}=7.68, \mathrm{p}<.001)$ compared to when asked about vaccine attitudes first $(\beta=-0.15, \mathrm{t}=3.55, \mathrm{p}<.001)$. This interaction indicates that making one's alignment with African American culture salient (by administering the acculturation scale before the vaccine attitudes scale) substantially increases the association between acculturation and reported vaccine attitudes - suggesting a causal link.

What mechanism, then, explains the underlying the relationship between acculturation and vaccine attitudes? We find that suspicion of the medical system was strongly positively correlated with negative vaccine attitudes $(\beta=0.34, \mathrm{t}=12.09, \mathrm{p}<.001)$ and negatively correlated with acculturation $(\beta=-0.52, t=17.86, p<.001)$ - and that controlling for suspicion of the medical system reduces the magnitude of the relationship between acculturation and negative vaccination attitudes by $64.0 \%$ (95\% confidence interval for indirect effect from mediation analysis using 1000 bootstrap samples, $[-.340,-.192])$. Conversely, the belief that there are racial disparities in healthcare treatment was not significantly correlated with negative vaccine attitudes $(\beta=-0.01$, $\mathrm{t}=0.30, \mathrm{p}=.77$ ).

Finally, we consider cognitive reflection and science knowledge. Cognitive reflection was not significantly related to negative vaccine attitudes $(\beta=-0.04, \mathrm{t}=1.23, \mathrm{p}=.220)$, while basic 
science knowledge was significantly negatively related to negative vaccine attitudes $(\beta=-0.09$, $\mathrm{t}=3.06, \mathrm{p}=.002)$. The relationship between acculturation and negative vaccine attitudes was

largely unchanged when adding both of these constructs into the model ( $\beta=-0.23$ without, $\beta=0.22$ with), and neither construct significantly interacted with acculturation (cognitive reflection: $\mathrm{t}=0.15, \mathrm{p}=.88$; science knowledge: $\mathrm{t}=1.24, \mathrm{p}=0.215$ ).

\section{Discussion}

Our findings show that there are substantial intragroup differences in attitudes towards COVID-19 vaccines among Black Americans. This approach of measuring intragroup differences enables the identification of minority subgroups who may be more likely to have concerns about getting vaccinated. For example, interventions that provide health information should consider specific perceptions which effect negative vaccine attitudes for specific subgroups. In particular, our results suggest that general suspicion of the healthcare system rather than the perception that the healthcare system discriminates against Blacks, or differences in science knowledge, or cognitive reflection - may be a key factor in the negative COVID-19 vaccination attitudes shown by Blacks who are most aligned with traditional African American culture. Our findings can thus be used to augment the current literature on messaging strategies that have been described for communicating COVID-19 information for racial minority populations, especially those underserved in research and those from groups who endure persistent racial inequity of health outcomes. ${ }^{15-19}$ Further, our findings can be used to design interventions that provide health information that targets the relevant factors for vaccine hesitancy in differing subgroups.

In the longer term, our work informs future investigations to understand intragroup differences for other groups with high hesitancy and enables addressing COVID-19 vaccination inequities by target messaging accordingly (e.g., rural conservative voters, rural older adults, etc.). Also, given that specific mechanisms between perceptions and intentions for specific health behaviors (e.g., primary care screenings) have not been described, ${ }^{20,21}$ our findings can help inform future investigations to describe pathways from perceptions (e.g., bias and discrimination) to health behaviors (e.g., cancer screening) to health outcomes (e.g., cancer treatment selection and outcomes).

\section{Materials and method}

We recruited N=997 Black Americans between February 19 and March 22021 to complete an online survey using the recruitment company Lucid. Participants completed a 9 item battery of attitudes towards the COVID-19 vaccine, which is our main dependent variable (alpha $=0.85$ ). They also completed the 47 item AAAS-R acculturation scale (scored by averaging the items within each subscale, and then averaging the 8 subscales), the 12 item Group-Based Medical Mistrust Scale (GBMMS), a 4 item science knowledge scale, and a 3 item Cognitive Reflection Test. Although prior work identified a three-factor solution for the 12 GBMMS items, factor analyzing our data (with promax rotation) showed only two factors: a factor capturing suspicion of the medical system onto which 8 items loaded heavily, and a factor capturing the sense that Blacks receive unequal treatment from the medical system onto which 4 items loaded 
heavily. Thus, we use these two factors in our analyses. All analyzes reported in the paper using linear regression with controls for age, age ${ }^{\wedge}$, gender (categorical), education (categorical 6-level variable), and socio-economic status (categorical 10-level variable eliciting using the MacArthur Scale of Subjective Social Status).

\section{Acknowledgements}

Members of the CovidAndRace team not listed among co-authors. We also would like to acknowledge support from the Canadian Institutes of Health Research.

\section{References}

1. Ogedegbe, G., et al., Assessment of Racial/Ethnic Disparities in Hospitalization and Mortality in Patients With COVID-19 in New York City. JAMA Network Open, 2020. 3(12): p. e2026881-e2026881.

2. Millett, G.A., et al., Assessing differential impacts of COVID-19 on black communities. Annals of Epidemiology, 2020. 47: p. 37-44.

3. Fisher, K.A., et al., Attitudes Toward a Potential SARS-CoV-2 Vaccine: A Survey of U.S. Adults. Ann Intern Med, 2020. 173(12): p. 964-973.

4. $\quad$ Momplaisir, F., et al., Understanding Drivers of Coronavirus Disease 2019 Vaccine Hesitancy Among Blacks. Clinical Infectious Diseases, 2021.

5. Klonoff, E.A. and H. Landrine, Revising and Improving the African American Acculturation Scale. Journal of Black Psychology, 2000. 26(2): p. 235-261.

6. Landrine, H. and E.A. Klonoff, The African American Acculturation Scale: Development, Reliability, and Validity. Journal of Black Psychology, 1994. 20(2): p. 104-127.

7. Osvold, L.L. and G.R. Sodowsky, Eating Attitudes of Native American and African American Women: Differences by Race and Acculturation. Explorations in Ethnic Studies, 1995. 18(2): p. 187-210.

8. Kimbrough, R.M., S.D. Molock, and K. Walton, Perception of social support, acculturation, depression, and suicidal ideation among African American college students at predominantly Black and predominantly White universities. 1996, Assn of American University Presses/Howard University Press: US. p. 295-307.

9. Klonoff, E.A. and H. Landrine, Distrust of Whites, Acculturation, and AIDS Knowledge Among African Americans. Journal of Black Psychology, 1997. 23(1): p. 50-57.

10. Klonoff, E.A. and H. Landrine, Acculturation and Cigarette Smoking Among African Americans: Replication and Implications for Prevention and Cessation Programs. Journal of Behavioral Medicine, 1999. 22(2): p. 195-204.

11. Schwartz, S.J., et al., Rethinking the concept of acculturation: implications for theory and research. The American psychologist, 2010. 65(4): p. 237-251.

12. Thompson, H.S., et al., The Group-Based Medical Mistrust Scale: psychometric properties and association with breast cancer screening. Preventive Medicine, 2004. 38(2): p. 209-218.

13. Hamoda, R.E., et al., Association of sociocultural factors with initiation of the kidney transplant evaluation process. American Journal of Transplantation, 2020. 20(1): p. 190-203.

14. Pennycook, G., et al., Beliefs about COVID-19 in Canada, the U.K., and the U.S.A.: A novel test of political polarization and motivated reasoning. 2021.

15. National Academies of Sciences Engineering and Medicine, Strategies for Building Confidence in the COVID-19 Vaccines. 2021, Washington, DC: The National Academies Press.

16. Schoch-Spana, M., et al. The Public's Role in COVID-19 Vaccination: Planning Recommendations Informed by Design Thinking and the Social, Behavioral, and Communication Sciences. 2020.

17. Best, A.L., et al., Institutional Distrust among African Americans and Building Trustworthiness in the COVID-19 Response: Implications for Ethical Public Health Practice. Journal of Health Care for the Poor and Underserved, 2021. 32(1): p. 90-98.

18. Viswanath, K., E.W.J. Lee, and R. Pinnamaneni, We Need the Lens of Equity in COVID-19 Communication. Health Commun, 2020. 35(14): p. 1743-1746. 
19. Chou, W.-Y.S. and A. Budenz, Considering Emotion in COVID-19 Vaccine Communication: Addressing Vaccine Hesitancy and Fostering Vaccine Confidence. Health Commun, 2020. 35(14): p. 1718-1722.

20. Ogedegbe, G., C.A. Mancuso, and J.P. Allegrante, Expectations of blood pressure management in hypertensive African-American patients: a qualitative study. Journal of the National Medical Association, 2004. 96(4): p. 442-449.

21. Benkert, R., et al., Ubiquitous Yet Unclear: A Systematic Review of Medical Mistrust. Behavioral Medicine, 2019. 45(2): p. 86-101. 